\title{
Photochemistry of the PAH pyrene in water ice: the case for ion-mediated solid-state astrochemistry
}

\author{
J. Bouwman ${ }^{1}$, H. M. Cuppen ${ }^{1}$, A. Bakker ${ }^{1}$, L. J. Allamandola ${ }^{2}$, and H. Linnartz ${ }^{1}$ \\ 1 Raymond and Beverly Sackler Laboratory for Astrophysics, Leiden Observatory, Leiden University, PO Box 9513, \\ 2300 RA Leiden, The Netherlands \\ e-mail: bouwman@strw. leidenuniv.nl \\ 2 NASA-Ames Research Center, Space Science Division, Mail Stop 245-6, Moffett Field, CA 94035, USA
}

Received 14 September 2009 / Accepted 9 November 2009

\begin{abstract}
Context. Icy dust grains play an important role in the formation of complex molecules in the interstellar medium (ISH). Laboratory studies have mainly focused on the physical interactions and chemical pathways in ices containing rather simple molecules, such as $\mathrm{H}_{2} \mathrm{O}, \mathrm{CO}, \mathrm{CO}_{2}, \mathrm{CH}_{4}$, and $\mathrm{CH}_{3} \mathrm{OH}$. Observational studies show that polycyclic aromatic hydrocarbons (PAHs) are also abundantly present in the ISM in the gas phase. It is likely that these non-volatile species also freeze-out onto dust grains and participate in the astrochemical solid-state network, but additional experimental PAH ice studies are largely lacking.

Aims. The study presented here focuses on a rather small PAH, pyrene $\left(\mathrm{C}_{16} \mathrm{H}_{10}\right)$, and aims to understand and quantify photochemical reactions of PAHs in interstellar ices upon vacuum ultraviolet (VUV) irradiation as a function of astronomically relevant parameters. Methods. Near UV/VIS spectroscopy is used to track the in situ VUV driven photochemistry of pyrene containing ices at temperatures ranging from 10 to $125 \mathrm{~K}$.

Results. The main photoproducts of VUV photolyzed pyrene ices are spectroscopically identified and their band positions are listed for two host ices, $\mathrm{H}_{2} \mathrm{O}$ and $\mathrm{CO}$. Pyrene ionization is found to be most efficient in $\mathrm{H}_{2} \mathrm{O}$ ices at low temperatures. The reaction products, triplet pyrene and the 1-hydro-1-pyrenyl radical are most efficiently formed in higher temperature water ices and in low temperature $\mathrm{CO}$ ice. Formation routes and band strength information of the identified species are discussed. Additionally, the oscillator strengths of $\mathrm{Py}, \mathrm{Py}^{+}$, and $\mathrm{PyH}^{-}$are derived and a quantitative kinetic analysis is performed by fitting a chemical reaction network to the experimental data.

Conclusions. Pyrene is efficiently ionized in water ice at temperatures below $50 \mathrm{~K}$. Hydrogenation reactions dominate the chemistry in low temperature $\mathrm{CO}$ ice with trace amounts of water. The results are placed in an astrophysical context by determining the importance of PAH ionization in a molecular cloud. We conclude that the rate of pyrene ionization in water ice mantles is comparable to the rate of photodesorption of $\mathrm{H}_{2} \mathrm{O}$ ice. The photoprocessing of a sample PAH in ice described in this manuscript indicates that PAH photoprocessing in the solid state should also be taken into account in astrochemical models.
\end{abstract}

Key words. astrochemistry - molecular processes - methods: laboratory - techniques: spectroscopic

\section{Introduction}

Strong infrared emission attributed to polycyclic aromatic hydrocarbons (PAHs) is characteristic of many galactic and extragalactic objects (Smith et al. 2007; Draine et al. 2007; Draine \& Li 2007; Tielens 2008). While this emission generally originates in optically thin, diffuse regions, PAHs should also be common throughout the dense interstellar medium. There, as with most other interstellar species in molecular clouds, PAHs condense out of the gas onto cold icy grain mantles, where they are expected to influence or participate in the chemistry and physics of the ice. While laboratory studies of interstellar ice analogs have shown that complex organic molecules are produced upon extended vacuum ultraviolet (VUV) photolysis (e.g., Briggs et al. 1992; Bernstein et al. 1995), the photoinduced processes occurring during the irradiation of PAH containing interstellar ice analogues have not yet been studied in detail. In optical, in situ studies of the photochemistry of naphthalene, 4-methylpyrene, and quatterylene containing water ice at $20 \mathrm{~K}$, Gudipati \& Allamandola (2003, 2006a,b) and Gudipati (2004) showed that these PAHs are readily ionized and stabilized within the ice, suggesting that trapped ions may play important, but overlooked roles in cosmic ice processes. Beyond this, there is little information about the VUV induced, in situ photochemistry and photophysics of PAH-containing water-rich ices.

Here, we describe a detailed study of the VUV-induced photochemistry that takes place within pyrene $\left(\mathrm{Py}\right.$ or $\left.\mathrm{C}_{16} \mathrm{H}_{10}\right)$ containing water ices (Py: $\left.\mathrm{H}_{2} \mathrm{O}=1: 10000-1: 5000\right)$. The present study is an extension of the study of Bouwman et al. (2009) in which the focus was on the new experimental setup and where the use of PAH ice spectra was discussed to search for solid-state features of PAHs in space. In this work, the focus is on a detailed characterization of the chemical processes taking place upon VUV irradiation, particularly as a function of ice temperature ranging from 25 to $125 \mathrm{~K}$. Additionally, measurements on Py:CO ices at $10 \mathrm{~K}$ were performed to elucidate the role of water in the reaction schemes and to clarify the formation routes of identified species. A similar study of three small PAHs is now underway to understand the general principles of $\mathrm{PAH} /$ ice photochemistry. This is part of an overall experimental program at the Sackler Laboratory for Astrophysics to study the fundamental processes of inter- and circumstellar ice analogues such as thermal (Acharyya et al. 2007) and photodesorption (Öberg et al. 2007b, 2009c), hydogenation reactions 
A\&A 511, A33 (2010)

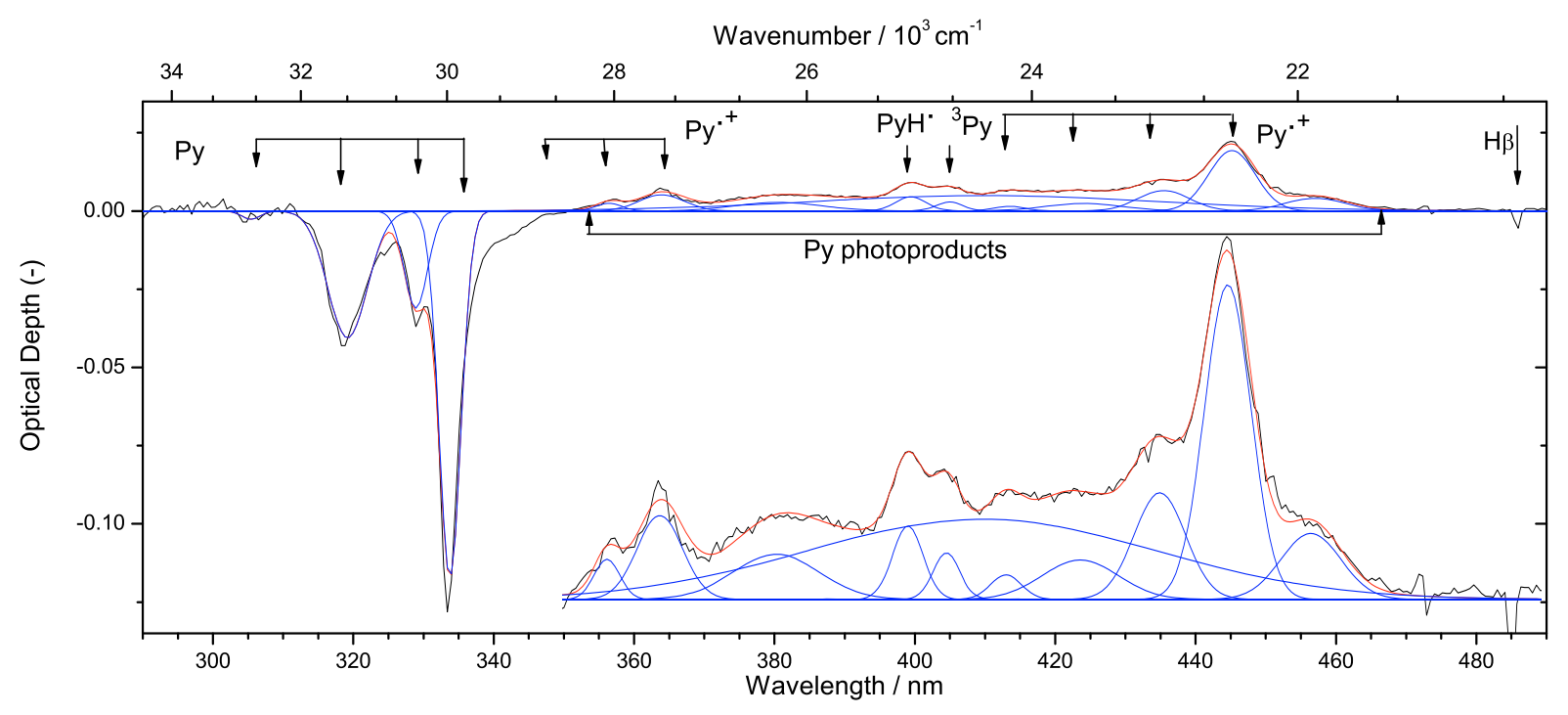

Fig. 1. The spectrum of a dilute pyrene: $\mathrm{H}_{2} \mathrm{O}$ ice after $900 \mathrm{~s}$ of VUV irradiation at $125 \mathrm{~K}$. The inset shows a blow-up of the pyrene photoproduct bands. Band assignments are discussed in Sect. 3. Note the broad feature ranging from about 350 to $470 \mathrm{~nm}$ which is indicated by a Gaussian fit. This is attributed to overlapping bands from individual pyrene photoproducts. Bands with negative optical depth indicate species destruction, those with positive optical depth show species formation. The blue bands are Gaussian profiles which co-add to the overall fit shown in red. Note the instrumental resolution indicated by the profile of the $\mathrm{H} \beta$ line at $486.1 \mathrm{~nm}$.

(Fuchs et al. 2009; Ioppolo et al. 2008), photochemistry (Öberg et al. 2009b), and physical interactions in interstellar ice analogues (Bouwman et al. 2007; Öberg et al. 2007a, 2009a).

The manuscript is organized as follows. The experimental technique is summarized in Sect. 2. Section 3 describes the $\mathrm{Py}: \mathrm{H}_{2} \mathrm{O}$ and $\mathrm{Py}: \mathrm{CO}$ ice photochemistry, the resulting products and their formation routes. The temperature-dependent photochemistry and derived reaction dynamics are described in Sect. 4 and astrochemical implications are discussed in Sect. 5. The main conclusions are summarized in Sect. 6.

\section{Experimental technique}

We use a new apparatus as described in Bouwman et al. (2009), which follows the photochemistry in kinetic mode during VUV irradiation by measuring the near UV-visible absorption spectra of an ice, providing "real-time" tracking of the reactants and photoproducts. Dilute $\mathrm{Py}: \mathrm{H}_{2} \mathrm{O}$ ice samples $(\sim 1: 10000-$ $\sim 1: 5000)$ and a Py:CO ice sample of comparable concentration are prepared by depositing the vapor from a pyrene sample heated to $40^{\circ} \mathrm{C}$ together with $\mathrm{H}_{2} \mathrm{O}$ vapor or $\mathrm{CO}$ gas onto a cold $\mathrm{MgF}_{2}$ window. The window is cooled to $10 \mathrm{~K}$ in the case of $\mathrm{CO}$ deposition or $25 \mathrm{~K}$ in the case of $\mathrm{H}_{2} \mathrm{O}$ deposition. The sample window is cooled by a closed cycle He refrigerator. Pyrene (Aldrich, 99\%) and CO (Praxair 99.999\%) are used as commercially available. Vapor from water, filtered through a milli-Q purification system and purified further by three freezepump-thaw cycles, is used. The sample window is mounted in a high-vacuum chamber $\left(P \approx 10^{-7} \mathrm{mbar}\right)$. The ice growth rate and thickness are determined with a HeNe laser by monitoring the thin-film interference fringes generated during deposition. Simultaneously, the amount of pyrene is tracked by measuring the integrated strength of the $S_{2} \leftarrow S_{0}$ neutral Py transition at $334 \mathrm{~nm}$. Deposition is typically stopped when the optical depth (OD) of Py approaches $\sim 0.15$.

The ice samples are photolyzed with the $121.6 \mathrm{~nm}$ Ly $\alpha$ $(10.6 \mathrm{eV})$ and the $160 \mathrm{~nm}$ molecular hydrogen emission bands (centered around $7.8 \mathrm{eV}$ ) generated by a microwave powered discharge in a flowing $\mathrm{H}_{2}$ gas with a vacuum ultraviolet flux of $\sim 10^{15}$ photons s $^{-1}$ (Muñoz Caro et al. 2002). This results in a photon flux of $\sim 10^{14}$ photons $\mathrm{cm}^{-2} \mathrm{~s}^{-1}$ at the sample surface (Öberg et al. 2009c).

Absorption spectra of VUV-photolyzed Py-containing ices are measured with a Xe-arc lamp serving as a white light source. Lenses and diaphragms direct the light through the ice sample along the optical axis determined by the HeNe laser beam after which it is focused onto the entrance slit of a $0.3 \mathrm{~m}$ spectrometer. A 150 lines $\mathrm{mm}^{-1}$ grating, blazed at $300 \mathrm{~nm}$, disperses the light onto a sensitive $1024 \times 256$ pixel CCD camera with 16 bit digitization. The camera is read out in vertical binning mode by a data acquisition computer that converts the data to absorbance spectra (OD $\left.=-\ln \left(I / I_{0}\right)\right)$. This configuration spans the 270 to $830 \mathrm{~nm}$ spectral range, which permits simultaneously monitoring of the behavior of the neutral Py parent molecule and photoproduct bands without any adjustment of the elements along the optical path. This is critical to obtaining reliable and reproducible baselines in measuring the optical spectra of ices. The spectral resolution is of the order of $0.9 \mathrm{~nm}$, which is more than sufficient to record broad solid-state absorption features.

The measurements described here were performed on various $\mathrm{H}_{2} \mathrm{O}$ :Py ice samples at 25, 50, 75, 100, and $125 \mathrm{~K}$. The CO ice experiments were carried out at $10 \mathrm{~K}$ to avoid matrix sublimation at higher temperatures. The sample temperature is maintained using a resistive heater with an accuracy of $\pm 2 \mathrm{~K}$. The measured spectra are converted into units of optical depth by using the spectrum of the freshly deposited, unphotolyzed ice at the appropriate temperature as a reference spectrum $\left(I_{0}\right)$. Recording a single spectrum typically takes about $5 \mathrm{~ms}$, and 229 spectra are generally coadded to improve the $\mathrm{S} / \mathrm{N}$ of a spectrum, producing one single spectrum every 10 seconds.

The optical configuration of the apparatus is such that spectra are recorded simultaneously with photolysis. Thus, the short spectral recording time permits us to monitor photoinduced changes on a roughly 10 -second time scale. Figure 1 shows the 290 to $490 \mathrm{~nm}$ spectrum of a Py: $\mathrm{H}_{2} \mathrm{O}$ ice at $125 \mathrm{~K}$ after $900 \mathrm{~s}$ of in situ VUV photolysis. Because the spectrum recorded before 
VUV irradiation is taken as a reference $\left(I_{0}\right)$, bands with positive OD values originate from species that are produced by photolysis, while the bands with negative OD correspond to the neutral pyrene that is lost upon photolysis. Comparing the Py and photoproduct absorption bands with the narrow $\mathrm{H} \beta$ lamp line at $486.1 \mathrm{~nm}$ shows that the instrumental resolution indeed far exceeds the ice band widths. The absolute wavelength calibration is accurate to within $\pm 0.5 \mathrm{~nm}$.

More than 1400 individual spectra are recorded and are reduced in a typical $4 \mathrm{~h}$ experiment. Spectra are individually baseline-corrected by fitting a second order polynomial through data points where no absorption occurs and subsequently subtracting the fit from the measured spectrum. Integrated absorbances of absorption features are calculated numerically for all spectra. These are corrected for the contributions of atomic hydrogen lines originating in the $\mathrm{H}_{2}$ discharge lamp. The data reduction software also allows us to plot correlation diagrams between integrated absorbances of different absorption features. All data handling and reduction is performed with LabView routines.

Integrated band areas are used, in conjunction with oscillator strengths $(f)$, to derive molecular abundances. The oscillator strength is converted to integrated absorbance $\left(\mathrm{cm}\right.$ molecule ${ }^{-1}$ ) using the conversion factor $8.88 \times 10^{-13}$ (Kjaergaard et al. 2000). The number of molecules per $\mathrm{cm}^{2}(N)$ is given by

$N=\frac{\int_{v_{1}}^{v_{2}} \tau \mathrm{d} v}{8.88 \times 10^{-13} f}$,

where $\tau$ is the optical depth and $v$ is the frequency in $\mathrm{cm}^{-1}$.

\section{Band assignments and band strength analysis}

The typical photolysis duration of about $4 \mathrm{~h}$ is the time required for nearly complete loss of the neutral pyrene vibrational progression at 334.0, 329.2, and $319.2 \mathrm{~nm}$. Irradiating the sample ices with VUV light produces a set of new absorption bands in the spectra, indicating active photochemistry. The band positions, $F W H M$, and assignments of the bands in the $\mathrm{Py}: \mathrm{H}_{2} \mathrm{O}$ ice at $25 \mathrm{~K}$ are listed in Table 1 . The bands appearing in the $\mathrm{Py}: \mathrm{CO}$ ice at $10 \mathrm{~K}$ are similar to those in the $\mathrm{Py}: \mathrm{H}_{2} \mathrm{O}$ ice at $25 \mathrm{~K}$, although, with slightly altered band positions and FWHM and with very different relative intensities (see also Table 1 ). Figure 1 presents a spectrum from the $125 \mathrm{~K} \mathrm{Py}: \mathrm{H}_{2} \mathrm{O}$ series. This figure illustrates production of the pyrene radical cation $\left(\mathrm{Py}^{+}\right)$, triplet pyrene ( $\left.{ }^{3} \mathrm{Py}\right), 1$-hydro-1-pyrenyl radical $\left(\mathrm{PyH}^{-}\right)$, and a broad underlying "residue" feature upon VUV irradiation. Additionally, a progression of distinct absorption features is found in the Py:CO experiment, which indicates the formation of the (reactive intermediate) HCO radical. The identifications of these species and their oscillator strengths are discussed below.

\subsection{Neutral pyrene bands}

As in Bouwman et al. (2009), the strong, negative bands peaking at $334.0 \mathrm{~nm}$ and weaker bands at 329.2 and $319.2 \mathrm{~nm}$ in the $\mathrm{H}_{2} \mathrm{O}$ ice (see Fig. 1), and at slightly shifted positions in the $\mathrm{CO}$ ice, are assigned to the ${ }^{1} B_{2 u} \leftarrow{ }^{1} A_{g}$ electronic transition of neutral pyrene $\left(\mathrm{S}_{2} \leftarrow \mathrm{S}_{0}\right)$ based on previous studies of pyrene in rare gas matrices (Vala et al. 1994; Halasinski et al. 2005). To study the chemistry in absolute number densities, a value of $f=0.33$ is adopted from the literature for the oscillator strength of pyrene (Bito et al. 2000; Wang et al. 2003). This value is used throughout this paper both for the $\mathrm{Py}: \mathrm{H}_{2} \mathrm{O}$ and $\mathrm{Py}: \mathrm{CO}$ experiments. Pure
Table 1. Band positions $\left(\lambda_{c}\right)$ and $F W H M$ in nm for pure pyrene ice at $10 \mathrm{~K}$, pyrene in $\mathrm{H}_{2} \mathrm{O}$ ice at $25 \mathrm{~K}$, pyrene in $\mathrm{CO}$ ice at $10 \mathrm{~K}$, and photoproduct bands for the $\mathrm{Py}: \mathrm{H}_{2} \mathrm{O}$ and $\mathrm{Py}: \mathrm{CO}$ UV processed ices.

\begin{tabular}{|c|c|c|c|c|c|c|}
\hline \multirow[t]{2}{*}{ Species } & \multicolumn{2}{|c|}{ Pyrene } & \multicolumn{2}{|c|}{ Pyrene: $\mathrm{H}_{2} \mathrm{O}$} & \multicolumn{2}{|c|}{ Pyrene:CO } \\
\hline & $\lambda_{c}$ & $F W H M$ & $\lambda_{c}$ & $F W H M$ & $\lambda_{c}$ & $F W H M$ \\
\hline \multirow[t]{3}{*}{$\operatorname{Py}{ }^{1} B_{2 u}$} & 312.7 & 7.1 & 319.2 & 6.5 & 319.4 & 7.5 \\
\hline & 325.3 & 10.0 & 329.2 & 3.2 & 329.2 & 2.3 \\
\hline & 341.5 & 14.0 & 334.0 & 4.4 & 334.3 & 4.1 \\
\hline \multirow[t]{3}{*}{$\mathrm{Py}^{+2} B_{1 u}$} & $\ldots$ & $\ldots$ & 363.2 & 3.6 & $\ldots^{a}$ & $\ldots^{a}$ \\
\hline & $\ldots$ & $\ldots$ & 354.0 & 6.5 & $\ldots^{a}$ & $\ldots^{a}$ \\
\hline & $\ldots$ & $\ldots$ & 344.9 & 6.2 &...$^{a}$ & $\ldots^{a}$ \\
\hline \multirow[t]{4}{*}{$\mathrm{Py}^{+2} A_{u}$} & ... & $\ldots$ & 445.6 & 6.6 & 445.3 & 7.8 \\
\hline & $\cdots$ & ... & 435.5 & 10.2 & $\ldots^{a}$ & $\ldots{ }^{a}$ \\
\hline & $\ldots$ & $\ldots$ & 423.0 & 12.2 &.$^{a}$ & $\ldots^{a}$ \\
\hline & 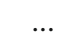 & $\ldots$ & 413.8 & 5.3 & $\ldots^{a}$ & $\ldots^{a}$ \\
\hline \multirow{4}{*}{$\begin{array}{l}\mathrm{Py}^{++2} B_{1 u} \\
\text { PyH }\end{array}$} & $\ldots$ & $\ldots$ & 490.1 & 10.0 & $\ldots^{a}$ & $\ldots^{a}$ \\
\hline & $\ldots$ & $\ldots$ & $399.4^{b}$ & $5.2^{b}$ & 400.5 & 4.2 \\
\hline & $\ldots$ & $\ldots$ &...$^{a}$ &...$^{a}$ & 392.5 & 6.7 \\
\hline & & & $\ldots^{a}$ & $\ldots^{a}$ & 378.4 & 15.7 \\
\hline \multirow{7}{*}{$\begin{array}{l}{ }^{3} \mathrm{Py}^{3} A_{g}^{-} \\
\mathrm{HCO}^{-2}{ }^{2}\end{array}$} & $\ldots$ & $\ldots$ & $405.0^{b}$ & $4.5^{b}$ & 406.2 & 4.8 \\
\hline & $\ldots$ & $\ldots$ & $\ldots$ & $\ldots$ & 513.4 & 17.5 \\
\hline & $\ldots$ & $\ldots$ & $\ldots$ & $\ldots$ & 535.3 & 12.5 \\
\hline & $\ldots$ & $\ldots$ & $\ldots$ & $\ldots$ & 556.3 & 14.5 \\
\hline & $\ldots$ & $\ldots$ & $\ldots$ & $\ldots$ & 583.0 & 16.8 \\
\hline & $\ldots$ & $\ldots$ & $\ldots$ & $\ldots$ & 604.9 & 10.0 \\
\hline & $\ldots$ & $\ldots$ & $\ldots$ & $\ldots$ & 639.2 & 15.1 \\
\hline
\end{tabular}

Notes. ${ }^{(a)}$ Absorption feature was too weak to perform an accurate fit; (b) features are too weak at $25 \mathrm{~K}$; the $125 \mathrm{~K}$ values are indicated.

pyrene ice measured at $10 \mathrm{~K}$ shows broader absorptions located at $341.5,325.3$, and $312.7 \mathrm{~nm}$ (see Table 1). We did not perform VUV experiments on the pure pyrene sample.

\subsection{Pyrene cation bands}

Positive bands at 363.2, 354.0, and $344.9 \mathrm{~nm}$ appear upon photolysis in the $\mathrm{Py}: \mathrm{H}_{2} \mathrm{O}$ experiments. This progression is assigned to the ${ }^{2} B_{1 u} \leftarrow{ }^{2} B_{3 g}$ vibronic transition of the pyrene cation $\left(\mathrm{Py}^{++}\right)$ in accordance with the proximity to the band positions reported by Vala et al. (1994) and Halasinski et al. (2005). This transition for $\mathrm{Py}^{++}$in $\mathrm{H}_{2} \mathrm{O}$ ice was reported in Bouwman et al. (2009). The ${ }^{2} B_{1 u} \leftarrow{ }^{2} B_{3 g}$ transition is too weak to be detected in the Py:CO experiment. A stronger $\mathrm{Py}^{+}$progression occurs at 445.6, 435.5, 423.0, and $413.8 \mathrm{~nm}$ in water ice. Of these bands, only the strongest at $445.3 \mathrm{~nm}$ is detectable in the irradiated Py:CO ice. This progression is assigned to the ${ }^{2} A_{u} \leftarrow{ }^{2} B_{3 g}$ transition of $\mathrm{Py}^{++}$. The much weaker absorption caused by the ${ }^{2} B_{1 u} \leftarrow{ }^{2} B_{3 g} \mathrm{Py}^{++}$ transition at $490.1 \mathrm{~nm}$ in $\mathrm{H}_{2} \mathrm{O}$ is again undetectable in $\mathrm{CO}$.

In these $\mathrm{H}_{2} \mathrm{O}$ and $\mathrm{CO}$ ice experiments, $\mathrm{Py}^{+}$formation is the result of direct single photon ionization of the neutral species, following:

$\mathrm{Py} \stackrel{\mathrm{VUV}}{\longrightarrow} \mathrm{Py}^{+}+\mathrm{e}^{-}$.

We emphasize that ionization in $\mathrm{Py}: \mathrm{H}_{2} \mathrm{O}$ ices is far more efficient than in Py:CO ices. Additional measurements on Py:CO: $\mathrm{H}_{2} \mathrm{O}$ mixtures indicate that the presence of $\mathrm{H}_{2} \mathrm{O}$ indeed enhances the ionization. Hence, it is possible that water contamination in the $\mathrm{CO}$ ice is responsible for the formation of some, if not all, of the cation species in the Py:CO experiment. The role of water contamination in $\mathrm{CO}$ ice will be discussed in more detail in Sect. 3.3. 


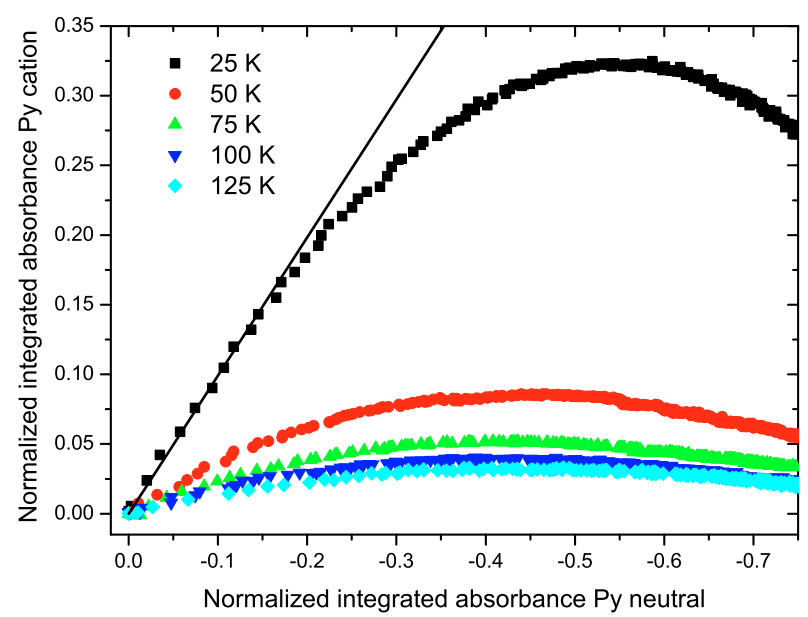

Fig. 2. Integrated absorbance of the $445.6 \mathrm{~nm} \mathrm{Py}^{+}$band growth plotted against the loss of the $334.0 \mathrm{~nm}$ Py band in the 25, 50, 75, 100, and $125 \mathrm{~K} \mathrm{Py}: \mathrm{H}_{2} \mathrm{O}$ ices. Ten seconds elapse between subsequent data points. Values are normalized to the maximum integrated absorbance of neutral pyrene. The straight line portion of these plots is used to determine the oscillator strength of $\mathrm{Py}^{+}$as described in Sect. 3.2.

Using baseline-corrected spectra as shown in Fig. 1, the photochemical evolution is tracked by integrating areas of bands produced by each species in every spectrum and plotting them as a function of photolysis time. The strongest $\mathrm{Py}^{+}$band at $\sim 445 \mathrm{~nm}$ is selected to track the number density evolution of this species. To put the kinetic analysis (Sect. 4) on a quantitative footing, we determine the oscillator strength of the $445 \mathrm{~nm} \mathrm{Py}^{+}$ band as follows. First, the integrated absorbance of the $445 \mathrm{~nm}$ $\mathrm{Py}^{+}$band is plotted versus that of the $334 \mathrm{~nm}$ Py band during the course of VUV photolysis at different ice temperatures. These graphs are shown in Fig. 2. It should be noted that there is a tight, linear behavior between the loss of neutral pyrene and growth of the pyrene cation during early photolysis times up to $100 \mathrm{~s}$ (the first 10 successive datapoints). Inspection of Fig. 2 shows that the slope is steepest and the ratio of the integrated absorbance of the $\mathrm{Py}^{+}$band to the Py band is optimum in the $25 \mathrm{~K}$ ice. Since no other photoproduct bands are evident during the linear correlation stage, we conclude that during this phase, neutral pyrene is converted solely into the cation as described previously for naphthalene and quaterrylene (Gudipati \& Allamandola 2006a). The straight-line portion, fitted through the first 10 data points of irradiation at $25 \mathrm{~K}$, is used to determine the oscillator strength of $\mathrm{Py}^{++}$. Given that the ratio of the $\mathrm{Py}^{++}$to the $334 \mathrm{~nm}$ Py band is 0.99 and the oscillator strength of this Py transition is 0.33 , the oscillator strength of the $443 \mathrm{~nm} \mathrm{Py}^{+}$band in water ice is also taken to be 0.33 . This conclusion is consistent with ab initio calculations on pyrene by Weisman et al. (2005). They calculated that the oscillator strength of the cation is only $\sim 2 \%$ stronger than that of the neutral species.

As described below, the photolysis of Py in water ices at higher temperatures produces other species in addition to the cation. This explains the different curves in Fig. 2.

\subsection{HCO bands in Py:CO}

VUV irradiation of a Py:CO ice also produces a vibrational progression ranging from $\sim 500$ to $650 \mathrm{~nm}$. As shown in Fig. 3, these absorption bands, located at 513.4, 535.3, 556.3, 583.0, 604.9, and $639.2 \mathrm{~nm}$, are assigned to the ${ }^{2} \mathrm{~A}^{\prime \prime}\left(0, v^{\prime \prime}, 0\right) \leftarrow \mathrm{X}^{2} A^{\prime}(0,0,0)$ $\mathrm{HCO}^{\circ}\left(v^{\prime \prime}=8-13\right)$ transitions based on band positions reported

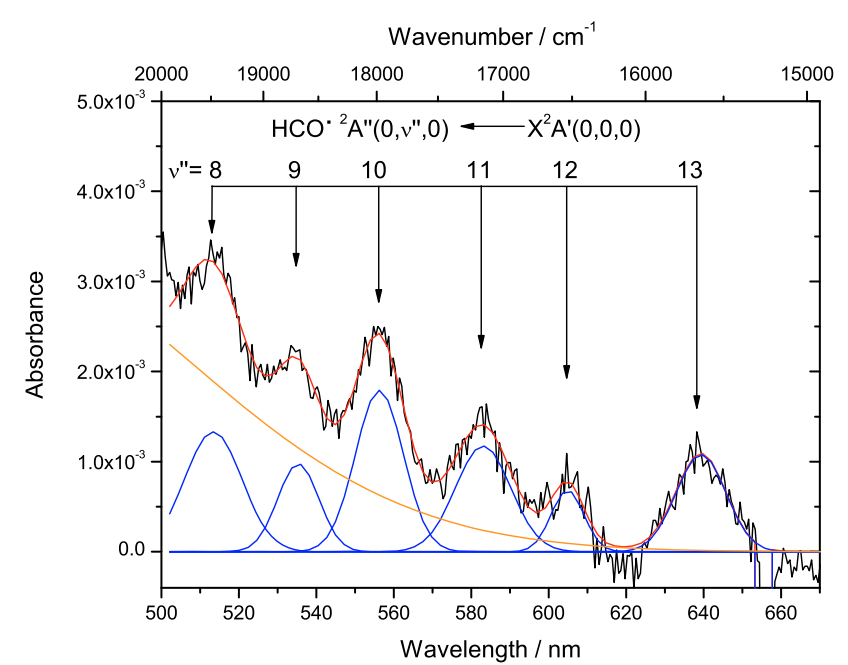

Fig. 3. Vibrational progression of $\mathrm{HCO}$ generated in a $\mathrm{Py}: \mathrm{CO}$ ice at $10 \mathrm{~K}$ after $600 \mathrm{~s}$ of VUV irradiation plotted together with a Gaussian fit (red) to the absorption spectrum (black). The individual Gaussians are shown in blue. The orange line indicates the red wing of the underlying broad absorption feature (Sect. 3.6).

by van Ijzendoorn et al. (1983). The clear HCO progression indicates a photolytic source of free $\mathrm{H}$ atoms in the ice. In addition, it confirms the ability of this setup to record small reactive intermediates in the ice.

A possible explanation of the source of $\mathrm{H}$ atoms is related to the experimental conditions. The experiments reported here are performed under high vacuum $\left(10^{-7} \mathrm{mbar}\right)$ conditions. Therefore, background $\mathrm{H}_{2} \mathrm{O}$ vapor has ample time to condense onto the sample window while cooling down and growing the ice sample. Water is well known to photodissociate upon VUV irradiation (e.g., Öberg et al. 2009c; Andersson \& van Dishoeck 2008) according to

$\mathrm{H}_{2} \mathrm{O} \stackrel{\text { VuV }}{\longrightarrow} \mathrm{OH}^{\cdot}+\mathrm{H}^{\circ}$

An experiment on VUV irradiation of a "pure" $\mathrm{CO}$ ice indicated that $\mathrm{HCO}$ is also efficiently produced in the absence of pyrene. Therefore, it is likely that water contamination is responsible for the production of $\mathrm{HCO}^{\text {via }}$

$\mathrm{H}^{\cdot}+\mathrm{CO} \rightarrow \mathrm{HCO}$.

Another possible formation route could be by means of VUVinduced hydrogen abstraction from pyrene. This pyrene photodissociation reaction, however, is unlikely to occur, since PAHs are generally highly photostable molecules.

\subsection{The $400 \mathrm{~nm}$ band carrier}

Another vibrational progression appears at 400.5, 392.5, and $378.4 \mathrm{~nm}$ in the CO ice experiments. As shown in Fig. 4, the $400.5 \mathrm{~nm}$ band dominates this progression. In contrast, a single band appears at $399.4 \mathrm{~nm}$ in the $\mathrm{Py}: \mathrm{H}_{2} \mathrm{O}$ ice upon VUV irradiation of the samples. The relative intensity of these bands varies with respect to the $\mathrm{Py}^{+}$bands. The $400 \mathrm{~nm}$ bands are more pronounced than the cation bands in the $\mathrm{H}_{2} \mathrm{O}$ ice only at high temperatures, whereas they are more pronounced in the low temperature $\mathrm{CO}$ ice.

Two additional measurements were performed to identify the carrier responsible for these transitions. A kinetic experiment was performed on non-VUV-irradiated Py:CO ice. This 


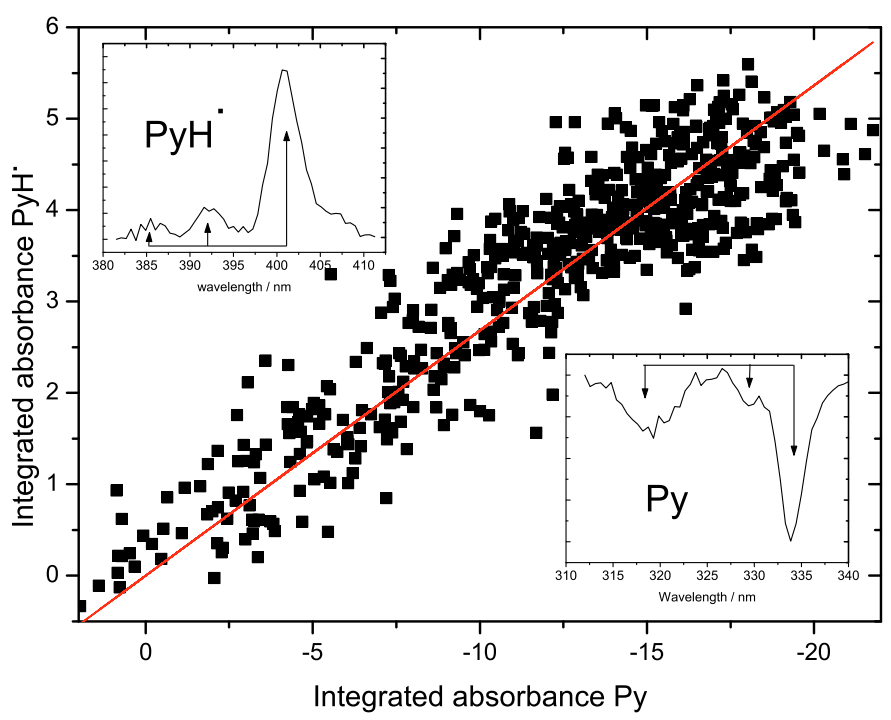

Fig. 4. Integrated absorbance of the $400 \mathrm{~nm} \mathrm{PyH}$ band growth plotted against the loss of the $334 \mathrm{~nm}$ Py band in a CO ice at $10 \mathrm{~K}$ after VUV irradiation is stopped. The straight line directly reflects the relative oscillator strength of both bands as described in Sect. 3.2. The two insets show the $\mathrm{PyH}^{\cdot}$ and $\mathrm{Py}$ vibrational progressions in a $\mathrm{CO}$ ice 90 min after photolysis is stopped.

ice showed no sign of pyrene ionization by the Xe-lamp, which is used as a spectroscopic light source. The production of $\mathrm{HCO}$. and the formation of the $400 \mathrm{~nm}$ band were not observed either. Subsequently, the ice was irradiated by the VUV source for $10 \mathrm{~min}$. The steady growth of the $400 \mathrm{~nm}$ band with VUV photolysis indicates that the species responsible for the $400 \mathrm{~nm}$ band is a product of the VUV processing of the ice. Moreover, when the VUV irradiation is stopped, the $400 \mathrm{~nm}$ band carrier continues to grow at the expense of the remaining neutral pyrene. This indicates that the chemical reaction leading to the formation of this species is not directly photon-dependent, but rather depends on the diffusion of a photoproduct. A similar experiment on a $\mathrm{Py}: \mathrm{H}_{2} \mathrm{O}$ ice at $25 \mathrm{~K}$ indicates that the same process also takes place in $\mathrm{H}_{2} \mathrm{O}$ ice. The detection of $\mathrm{HCO}^{-}$radicals in the ice and the inherent presence of free photolytic $\mathrm{H}$ atoms, implies that the growth of the vibrational progression starting at $\sim 400 \mathrm{~nm}$ could be the result of the reaction of pyrene with diffusing $\mathrm{H}$ atoms

$\mathrm{Py}+\mathrm{H} \rightarrow \mathrm{PyH}$.

This assignment to the 1-hydro-1-pyrenyl radical ( $\left.\mathrm{PyH}^{-}\right)$is supported by other experimental studies (Okada et al. 1976, 1980), where progressions at similar band positions are observed upon (laser) flash photolysis.

In contrast to the $\mathrm{Py}: \mathrm{H}_{2} \mathrm{O}$ experiments where pyrene is also efficiently ionized, the experiment on $\mathrm{PyH}^{-}$formation in $\mathrm{CO}$ shows no sign of other reaction products. The integrated absorbance of the growing $\mathrm{PyH}^{-}$transition is plotted versus the integrated absorbance of the diminishing neutral in Fig. 4. Growth is tracked over a duration of more than $1.5 \mathrm{~h}$. Since there is a oneto-one conversion of $\mathrm{Py}$ to $\mathrm{PyH}$ in the Py: $\mathrm{CO}$ ice (Eq. (5)), as described in Sect. 3.2, we derive an oscillator strength of 0.089 for this species by fitting a line through the correlating absorbances in Fig. 4.

\subsection{The $405 \mathrm{~nm}$ band carrier}

Besides the $\mathrm{Py}^{+}$and the $\mathrm{PyH}^{*}$ bands, another distinct absorption is found in the spectra of VUV irradiated ices. This feature is located at $405.0 \mathrm{~nm}$ in the $\mathrm{Py}: \mathrm{H}_{2} \mathrm{O}$ and at $406.2 \mathrm{~nm}$ in the $\mathrm{Py}: \mathrm{CO}$ experiment. In our previously published paper on low temperature $\mathrm{Py}: \mathrm{H}_{2} \mathrm{O}$ ice, we tentatively assigned this absorption to a negative ion, $\mathrm{Py}^{-}$or $\mathrm{PyO}^{-}$(Bouwman et al. 2009). The experiments on Py:CO ices presented here enable us to exclude this assignment because of the nearly absent $\mathrm{Py}^{++}$transitions. Firstly, $\mathrm{Py}^{-}$is ruled out because a much stronger second $\mathrm{Py}^{-}$absorption band, expected at $490 \mathrm{~nm}$ (Montejano et al. 1995), is absent in our Py:CO experiment. Secondly, $\mathrm{PyO}^{-}$is also ruled out, because it should exhibit absorption bands down to $350 \mathrm{~nm}$ (Milosavljevic $\&$ Thomas 2002), bands that are also absent in the Py:CO experiment. Additionally, in our previous paper we assumed that $\mathrm{PyO}^{-}$ was a product of $\mathrm{PyOH}$. The formation of $\mathrm{PyO}^{-}$is also unlikely in the absence of $\mathrm{PyOH}$ absorption in these experiments, as discussed below.

The absorption at $405 \mathrm{~nm}$ does not correlate with that of the cation, nor with the $\mathrm{PyH}$ band. The band only appears during photolysis and hence is characterized as a VUV-photon-related product. From the literature, it is known that a pyrene triplettriplet $\left({ }^{3} A_{g}^{-} \leftarrow{ }^{3} B_{2 u}^{+}\right)$transition is expected at this wavelength upon laser excitation of pyrene in solution which populates the lowest member of the triplet manifold (e.g., Hsiao \& Webber 1992; Langelaar et al. 1970). For the $405 \mathrm{~nm}$ band to originate from this triplet-triplet transition, the lowest level must be populated and remain so with a long enough lifetime to allow absorption to the ${ }^{3} A_{g}^{-}$level. In the ice experiments reported here, there are a number of possible routes for pumping the ${ }^{3} \mathrm{Py}$ state. The most obvious route is by means of photoexcitation followed by intersystem crossing

${ }^{1} \mathrm{Py} \stackrel{\text { vuV }}{\longrightarrow}{ }^{1} \mathrm{Py}^{*} \stackrel{\text { isc }}{\longrightarrow}{ }^{3} \mathrm{Py}$.

Triplet formation is found to decrease with decreasing temperature in ethanol ice (Stevens et al. 1967). This translates to our experiment in a nearly absent $405 \mathrm{~nm}$ band in the low temperature $\mathrm{Py}: \mathrm{H}_{2} \mathrm{O}$ experiment, because of the high $\mathrm{Py}^{+}$formation efficiency. In the high temperature $\mathrm{H}_{2} \mathrm{O}$ ice experiments, on the other hand, the $405 \mathrm{~nm}$ absorption is much stronger because pyrene is available.

In the $\mathrm{CO}$ ice, on the other hand, where $\mathrm{Py}^{+}$production is low, formation of the $405 \mathrm{~nm}$ band carrier appears to be very efficient at low temperatures. The production of the $405 \mathrm{~nm}$ band carrier requires VUV photons to be initiated. The pumping of the ${ }^{3}$ Py state can again occur by means of Eq. (6). Moreover, $\mathrm{CO}$ has a dipole allowed electronic transition in the VUV. Hence, speculating, pumping of the ${ }^{3} \mathrm{Py}$ state by collisional de-excitation of $\mathrm{CO}$ molecules exited by the VUV radiation provides a reaction path of

$\mathrm{CO} \stackrel{\mathrm{vuV}}{\longrightarrow} \mathrm{CO}^{*}$,

followed by

$\mathrm{CO}^{*}+{ }^{1} \mathrm{Py} \rightarrow{ }^{3} \mathrm{Py}+\mathrm{CO}$.

In summary, while we cannot identify the carrier of the $405 \mathrm{~nm}$ band, the ${ }^{3} A_{g}^{-} \leftarrow{ }^{3} B_{2 u}^{+}$transition seems a plausible explanation.

\subsection{Broad absorption feature}

Finally, besides the narrower bands reported in the previous sections, we discuss a broad underlying feature extending from 
about 350 to $570 \mathrm{~nm}$, which grows upon photolysis in all cases. This band probably comprises overlapping bands caused by a number of $\mathrm{Py} / \mathrm{H}_{2} \mathrm{O}$ or $\mathrm{Py} / \mathrm{CO}$ photoproducts. Part of this $\mathrm{Py}-$ residue feature remains even after warming up the sample window to room temperature, whereas all other features disappear at the water desorption temperature.

As discussed above, the very broad feature must be produced by a variety of similar but distinct photoproducts, all containing the pyrene chromophore. Mass spectral analysis of the species produced by the VUV photolysis of a few other PAHs in water ice show that the parent $\mathrm{PAH}$ is not destroyed but that $\mathrm{OH}, \mathrm{O}$, and $\mathrm{H}$ are added to some of the edge carbon atoms (Bernstein et al. 1999). Given the multiplicity of the side sites on pyrene that can undergo substitution, it is likely that the photoproducts produced in the experiments reported here are multiply substituted, rather than singly substituted. Thus, it is possible that a mixture of related but distinct $\mathrm{Py}-\mathrm{X}_{n}$ species, where $\mathrm{X}$ may be $\mathrm{H}, \mathrm{OH}$, or $\mathrm{O}$, produce the broad band.

In our previous work, we reported the production of a clear and reproducible $\mathrm{PyOH}$ band at $344.9 \mathrm{~nm}$ in a low temperature $\mathrm{H}_{2} \mathrm{O}$ ice (Bouwman et al. 2009). The results presented here do not show evidence of this absorption feature. However, in some instances the absorption was detected upon irradiation or warm-up of the ice. The irregular appearance of the $\mathrm{PyOH}$ absorption feature in these experiments indicates that the formation of this species is highly sensitive to the sample's physical parameters, i.e., structure of the ice, temperature, and concentration. One possible explanation is that in the previously reported experiments, the Py concentration was not controlled and those experiments sampled a very different ice concentration and, by implication, physical ice structure. While we do not have a solution for this discrepancy, we emphasize that both measurement series have been fully reproducible over many independent experiments for periods of months. An experimental program to investigate the role of the $\mathrm{PAH}: \mathrm{H}_{2} \mathrm{O}$ concentration on ice photochemistry is underway.

\section{4. $\mathrm{Py}: \mathrm{H}_{2} \mathrm{O}$ ice photochemistry at different temperatures}

Figure 5 shows the spectral evolution of two different $\mathrm{Py}: \mathrm{H}_{2} \mathrm{O}$ samples at different temperatures. The top frame presents the 280 to $540 \mathrm{~nm}$ spectra of the $100 \mathrm{~K}$ Py: $\mathrm{H}_{2} \mathrm{O}$ ice after $0,20,40$, 80 , and $160 \mathrm{~s}$ of in situ photolysis and the bottom frame the corresponding spectra for the $25 \mathrm{~K}$ ice. These spectra are snapshots of the more than one thousand spectra collected during $4 \mathrm{~h}$ of photolysis. They illustrate the rapid changes that occur during the early stages in the photochemistry of these ices and the major differences in reaction products at different temperatures.

To probe the VUV-driven photophysics and reaction dynamics for a set of selected temperatures, the production and depletion of species was tracked as a function of irradiation time. To this end, the Py 334 nm, Py ${ }^{+} 445$ nm, and $\mathrm{PyH}^{\circ} 400 \mathrm{~nm}$ bands were integrated for every spectrum. The spectra in Figs. 1 and 5 show that it is rather straightforward to determine the boundaries needed to integrate these bands. We estimate that the uncertainty in most of these band areas is of the order of $10 \%$.

The integrated absorbances of the neutral $\mathrm{Py}$, strongest $\mathrm{Py}^{++}$, and $\mathrm{PyH}^{\circ}$ bands in $\mathrm{H}_{2} \mathrm{O}$ ice at temperatures of $25,50,75,100$, and $125 \mathrm{~K}$ are plotted versus photolysis time (VUV fluence) in Fig. 6. The spectra in Fig. 5 and photochemical behavior in Fig. 6 show that, upon photolysis, neutral pyrene loss is immediate and rapid. The initial growth of $\mathrm{Py}^{+}$mirrors the rapid, initial loss of Py. However, while Py steadily decreases, and several other Py

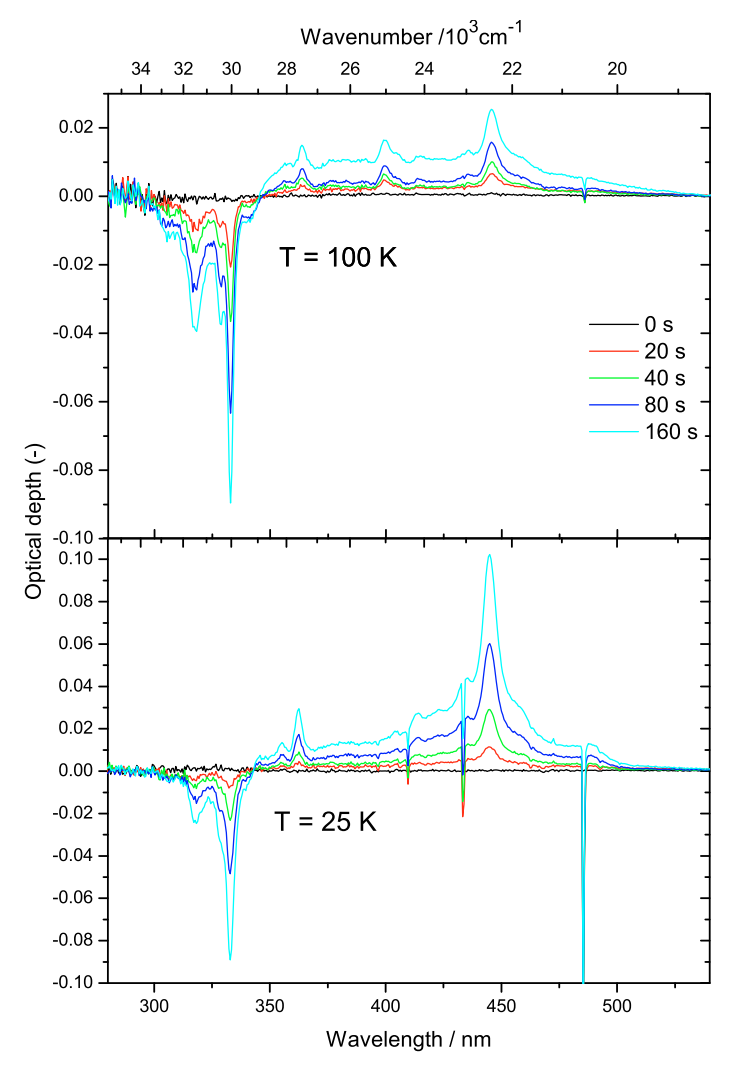

Fig. 5. The VUV-induced spectroscopic changes in $\mathrm{Py}: \mathrm{H}_{2} \mathrm{O}$ ice for two different temperatures as a function of photolysis time. Comparing the spectra from the $25 \mathrm{~K}$ ice (bottom) with those of the $100 \mathrm{~K}$ ice (top) shows the critical role that temperature plays in determining photochemical pathways in a PAH-containing ice. In the $25 \mathrm{~K}$ ice, cation formation is favored over production of the pyrene residue and the 400 and $405 \mathrm{~nm}$ band carriers. The opposite holds for the $100 \mathrm{~K}$ ice.

photoproduct bands increase during some $4 \mathrm{~h}$ of photolysis, the production of $\mathrm{Py}^{+}$reaches a maximum and then slowly diminishes. From Fig. 6, one can clearly see that ionization of pyrene is most efficient in the low temperature ice. Formation of $\mathrm{PyH}$, on the other hand, is far more efficient at higher temperatures.

For comparison, the integrated absorbances for the irradiated $\mathrm{Py}: \mathrm{CO}$ ice are plotted as a function of time in the right bottom frame of Fig. 6. It should be noted that the $\mathrm{PyH}^{\circ}$ band is multiplied by a factor of 10 in the Py:CO experiment, compared to a factor of 20 in the $\mathrm{Py}: \mathrm{H}_{2} \mathrm{O}$ experiment. The $\mathrm{PyH}^{\circ}$ band is clearly more prominent in the $\mathrm{CO}$ ice experiment than in the $\mathrm{H}_{2} \mathrm{O}$ ice experiments. The $\mathrm{Py}^{+}$signal on the other hand is negligible. This indicates that the $\mathrm{H}_{2} \mathrm{O}$ ice plays a role in ion formation and stabilization.

To place this behavior on a quantitative footing, the integrated areas for the $\mathrm{Py}$ and $\mathrm{Py}^{\cdot+}$ bands are converted to number densities using Eq. (1). Here, an oscillator strength of 0.33 is used for the $334 \mathrm{~nm}$ Py bands. The values used for the oscillator strengths of the $\mathrm{Py}^{++}$and $\mathrm{PyH}^{-}$bands are 0.33 and 0.089 , respectively, as determined in Sects. 3.2 and 3.4. Perusal of Fig. 6 shows that Py behaves similarly in all of the ices considered here. Regardless of temperature, its signal drops quickly with the onset of irradiation and continues to diminish with ongoing photolysis. Likewise, $\mathrm{Py}^{++}$grows rapidly with initial photolysis but peaks after a relatively short time interval corresponding to a fluence of roughly $8 \times 10^{16}$ photons and then drops continuously. While the $\mathrm{Py}^{+}$growth and loss curves resemble each other, cation production efficiency is strongest in the $25 \mathrm{~K}$ ice. 


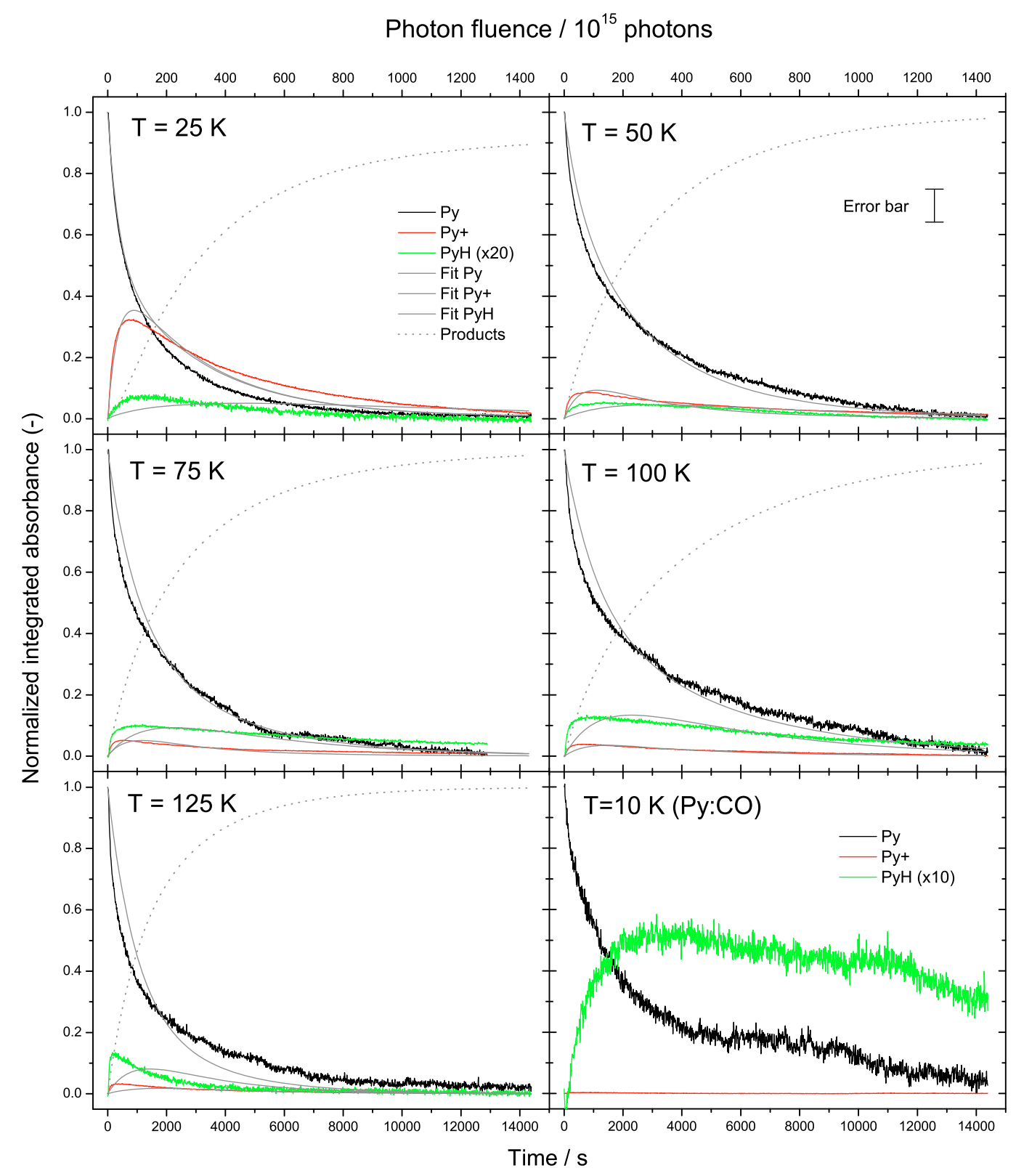

Fig. 6. The integrated absorbance of the $\mathrm{Py} 334 \mathrm{~nm}, \mathrm{Py}^{+} 445 \mathrm{~nm}$, and $\mathrm{PyH} 400 \mathrm{~nm}$ bands as a function of VUV irradiation in $\mathrm{Py}_{2} \mathrm{H}_{2} \mathrm{O}$ ices at 25 , 50, 75, 100, and $125 \mathrm{~K}$ and a Py:CO ice at $10 \mathrm{~K}$ plotted together with the fits (grey lines) described in Sect. 4. Integrated absorbance values are scaled and normalized to the initial value for the Py signal. The $\mathrm{PyH}$ feature is multiplied by a factor of 20 for the $\mathrm{Py}: \mathrm{H}_{2} \mathrm{O}$ experiments and by a factor of 10 for the Py:CO experiment. The approximate, overall growth of the total Py photoproduct band $\left(\mathrm{P}_{1}+\mathrm{P}_{2}+\mathrm{P}_{3}\right)$ is also shown (dotted line).

This efficiency remains of the same order at even lower temperatures (not shown here). The photolysis time required for the cation to reach a maximum shortens with increasing temperature. The $\mathrm{PyH}$ band contribution is minor with respect to the $\mathrm{Py}^{+}$band for ices below $50 \mathrm{~K}$. This reverses between 50 and $75 \mathrm{~K}$, suggesting that there is a change in the dominant $\mathrm{Py}: \mathrm{H}_{2} \mathrm{O}$ ice photochemical channel in this temperature range.

A kinetic analysis of the plots in Fig. 6 is carried out using the reaction scheme indicated in Fig. 7. Here, $k_{11}$ is the photoionization rate of $\mathrm{Py}$ to $\mathrm{Py}^{+}, k_{12}$ the electron-ion recombination rate of $\mathrm{Py}^{+}, k_{21}$ the production rate of the $\mathrm{PyH}^{-}$feature, and $k_{22}$ the rate of the reverse reaction of $\mathrm{PyH}$ to $\mathrm{Py}$. The rates designated $k_{1}, k_{2}$, and $k_{3}$ are the production rates for the different products that comprise the Py-residue band. The oscillator strengths for the $\mathrm{Py}^{+}$and $\mathrm{PyH}^{-}$bands are also fitted, but are restricted to remain within $\pm 10 \%$ of the experimentally determined values of 0.33 and 0.089 . All reactions are assumed to be first order in the reactant. The relative abundances of "free or solvated electrons" and $\mathrm{O}, \mathrm{H}$, and $\mathrm{OH}$ radicals in the ice are not considered.

The fits to the growth and decay curves are included in Fig. 6 and the temperature dependence of the derived rate constants is presented in Fig. 8. The agreement between the fit and the experimental data in terms of curve shape and absolute intensity is good. The fitted oscillator strengths of the $\mathrm{Py}^{-+}$and $\mathrm{PyH}^{\circ}$ bands amount to 0.31 and 0.082 , respectively, and hence do not deviate much from the experimentally determined values.

The graph in Fig. 8 indicates that the Py photoionization rate $\left(k_{11}\right)$ drops rapidly between 25 and $50 \mathrm{~K}$. The electron recombination rate $\left(k_{12}\right)$ decreases only slightly, if at all, within the errors over the entire temperature range. As mentioned above, 


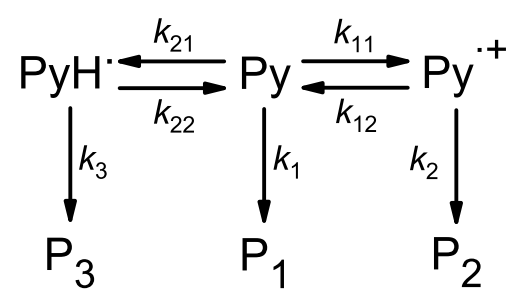

Fig. 7. Reaction scheme used to fit the experimental data.

the production of the $\mathrm{PyH}$ becomes more important at higher temperatures. Its formation rate $\left(k_{21}\right)$ is low in all ices up to $50 \mathrm{~K}$ $\left(<4.4 \times 10^{-5}\right)$, but jumps to $>1 \times 10^{-4}$ in the ices with temperatures of $75 \mathrm{~K}$ and higher. The back channel from $\mathrm{PyH}$ to $\mathrm{Py}$, $k_{22}$, also shows a temperature dependence. It increases almost linearly in going from cold to warm ices. The formation rate of a photoproduct produced directly from Py $\left(k_{1}\right)$ also seems to jump at $50 \mathrm{~K}$. The formation rate of products originating in the $\mathrm{Py}^{+}$species, on the other hand, seems to lower with increasing temperature. Finally, the rate of product formation from the $\mathrm{PyH}^{\circ}$ channel is low throughout the entire temperature range. The jump in rate of the formation of $\mathrm{P}_{1}$ and $\mathrm{PyH}^{-}$with temperature probably reflects the diffusion barrier of radical species $\left(\mathrm{H}^{\circ}\right.$ and $\mathrm{OH}^{\circ}$ ) in the ice.

Since published studies of the processes induced by the photolysis of other $\mathrm{PAH}: \mathrm{H}_{2} \mathrm{O}$ ices are limited, not much information is available with which to compare these results. While, to the best of our knowledge, there are no reports of the photochemistry that takes place as a function of ice temperature or of longterm fluence, the VUV photochemistry of the PAHs naphthalene, 4-methylpyrene (4MP), and quatterrylene in water ice at $10 \mathrm{~K}$ has been studied (Gudipati \& Allamandola 2003; Gudipati 2004; Gudipati \& Allamandola 2006a,b). The results obtained are in good agreement with the low temperature $(25 \mathrm{~K})$ case reported here. Namely, the parent PAH is easily and efficiently ionized, by quantitative conversion of the neutral species to the cation form. The focus of the earlier studies was on cation production and stabilization and not on long-duration photolysis experiments. In their study of 4MP: $\mathrm{H}_{2} \mathrm{O}(1:>500)$ ice at $15 \mathrm{~K}$, Gudipati \& Allamandola (2003) utilized a reaction scheme similar to that on the right half of that presented in Fig. 7. Table 2 compares the reaction rates that they determined with those of the $25 \mathrm{~K}$ ice reported here. Except for the production of $\mathrm{P}_{2}$, which differs by one order of magnitude, there is very good agreement between the rate constants for each step in the two experiments.

The growth and decay curves in Fig. 6, taken together with the temperature dependence of the reaction rates in Fig. 8, show that the VUV-driven PAH photochemistry depends strongly on ice temperature. The influence of the ice morphology on this chemistry was also investigated, to understand the origin of the temperature dependence. An experiment on an ice deposited at $25 \mathrm{~K}$, annealed to $125 \mathrm{~K}$, and subsequently cooled to $25 \mathrm{~K}$ before photolysis, showed that the ionization rate and efficiency are similar to that of an unannealed ice. Apparently, it is not the morphology but the temperature of the ice that primarily determines which process dominates. We discriminate between two temperature regimes. One governed by ion-mediated processes that dominate at $25 \mathrm{~K}$ and slightly higher temperatures, and a second, presumably radical-driven regime, that becomes increasingly more important at higher temperatures.
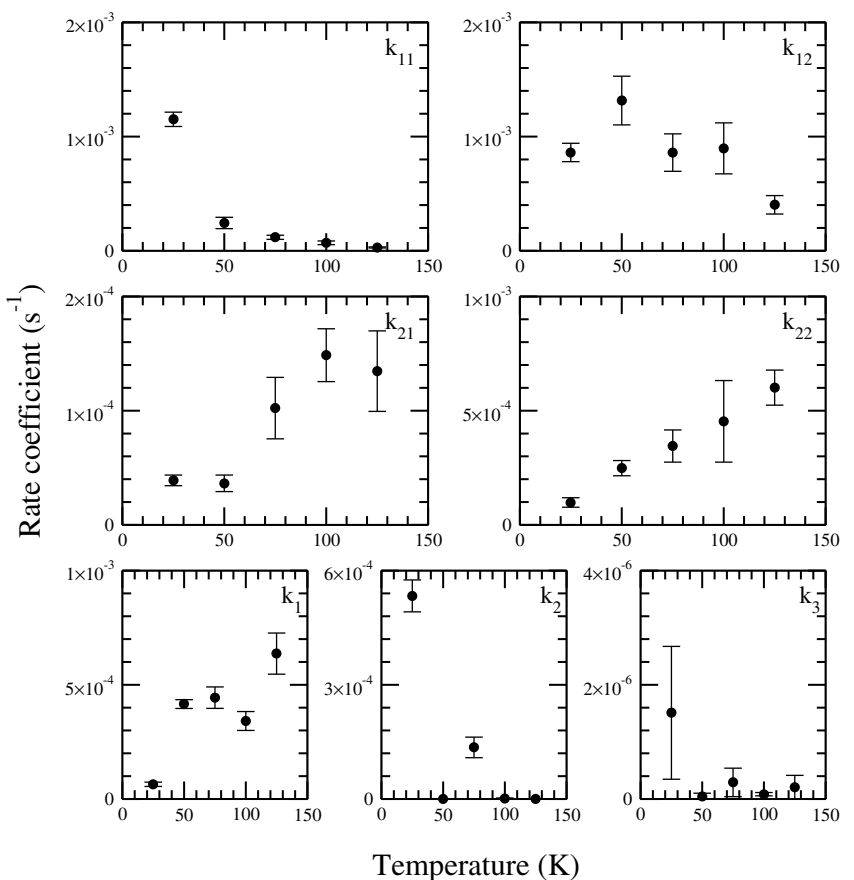

Fig. 8. Parameters $\left(k_{\mathrm{nm}}\right)$ as a function of temperature resulting from fitting the reaction scheme (Fig. 7) to the kinetic experiments (Fig. 6). All rates are indicated in $\mathrm{s}^{-1}$.

Table 2. The reaction rates for the VUV photolysis of $\mathrm{Py}: \mathrm{H}_{2} \mathrm{O}$ $(\sim 1: 5000)$ ice at $25 \mathrm{~K}$ compared to those for 4-methylypyrene: $\mathrm{H}_{2} \mathrm{O}$ (1:>500) ice at $15 \mathrm{~K}$ (Gudipati \& Allamandola 2003).

\begin{tabular}{|c|c|c|c|}
\hline Rate & $\begin{array}{c}\text { This work } \\
\left(\mathrm{s}^{-1}\right)\end{array}$ & $\begin{array}{l}\text { Photon rate } \\
\left(\mathrm{cm}^{2} \text { photon }^{-1}\right)\end{array}$ & $\begin{array}{c}\text { Gudipati (2003) } \\
\left(\mathrm{s}^{-1}\right)\end{array}$ \\
\hline $\mathrm{V} \stackrel{\mathrm{VUV}}{\longrightarrow} \mathrm{Py}^{++}$ & \pm 0.1 & $1.2 \times 10^{-17}$ & $1.3 \times 10^{-3}$ \\
\hline $\mathrm{Py}^{+}+\mathrm{e}^{-} \rightarrow \mathrm{Py}\left(k_{12}\right)$ & $(9 \pm 2$ & $9 \times 10^{-18}$ & $8 \times 10^{-4}$ \\
\hline $\mathrm{Py} \rightarrow \mathrm{P}_{1}$ & $(5 \pm 1) \times 10^{-5}$ & $\ldots$ & $4 \times 10^{-5}$ \\
\hline $\mathrm{Py}^{++} \rightarrow \mathrm{P}_{2}$ & $(5 \pm 1) \times 10^{-4}$ & $\ldots$ & $5 \times 10^{-5}$ \\
\hline
\end{tabular}

Notes. ${ }^{(a)}$ Photon rates are indicated only for reaction channels which are dominated by photon processes.

\section{Astrochemical implications}

As shown in the previous sections, ionization and chemistry of a rather small $\mathrm{PAH}$ species, pyrene, trapped in $\mathrm{H}_{2} \mathrm{O}$ ice turns out to be very efficient in a laboratory setting. Here, we extend these findings to interstellar conditions, with the aim of including the calculated rates in astrochemical models. For this, it is crucial to distinguish pure photochemical processes from diffusion, since the latter will be highly dependent on the number density of radicals and electrons in the ice. As mentioned in the previous section, the photoionization of Py is probably a single-photon process, whereas protonation of Py and the electron recombination of $\mathrm{Py}^{+}$are the results of both VUV photolysis and diffusion. The mechanism for $\mathrm{PyH}^{-}$deprotonation is unclear, since it can proceed by means of either VUV processing or through hydrogen abstraction by diffusing species. Diffusion of radicals through the ice is a thermally activated process and will therefore increase with temperature. Recombination, however, is largely temperature-independent in our experiments, indicating that the rate of $\mathrm{Py}^{\cdot+}$ recombination is not dominated by the diffusion of electrons in the ice. If Py+ loss occurs by means of electron recombination and not $\mathrm{Py}^{\cdot+}$ reaction with $\mathrm{H}_{2} \mathrm{O}$ or one of its 
photoproducts, the electron most likely originates from the initial photoionization event after which electrons remain in the vicinity of the recombining $\mathrm{Py}^{++}$species. Hence, this local process can be, although indirectly, regarded as a single-photon process.

The rates of protonation of $\mathrm{Py}$ and deprotonation of $\mathrm{PyH}$ show a temperature dependence and the importance of diffusion can therefore not be excluded. This makes it harder to directly translate the rates $\left(\mathrm{s}^{-1}\right)$ into photon rates $\left(\mathrm{cm}^{2}\right.$ photon $\left.{ }^{-1}\right)$. However, we can determine astrochemical photon rates for both ionization and recombination of pyrene in interstellar $\mathrm{H}_{2} \mathrm{O}$ ice (see Table 2).

Now, to translate this to the astrochemical situation and with other processes, we assume that PAHs generally have an ionization rate similar to that of pyrene. How do ionization and chemistry compare with other processes such as the photodesorption of the icy grain mantle, in which the PAHs are embedded? To exemplify this, the rate of ionization of a PAH in water ice at $25 \mathrm{~K}$ (in photon ${ }^{-1}$ ) is calculated anywhere in a dense cloud where $A_{V}=3$ and compared with the VUV photodesorption rate of $\mathrm{H}_{2} \mathrm{O}$ derived by Öberg et al. (2009c). It is well established that the onset of ice formation occurs in clouds with an edgeto-edge (through the cloud) magnitude of $A_{V}=3$ (e.g. Whittet et al. 2001). Thus, inside our hypothetical dense cloud at $A_{V}=3$ (from cloud edge to within the cloud), ices are present.

The experimentally determined $\mathrm{PAH}$ ionization rate in $\mathrm{H}_{2} \mathrm{O}$ at $25 \mathrm{~K}$, normalized to the total amount of deposited PAH is given by

$k_{11}=\frac{\mathrm{d} \frac{\left[\mathrm{PAH}^{+}\right]}{[\mathrm{PAH}]_{0}}}{\mathrm{~d} t}=10^{-3} \mathrm{~s}^{-1}$.

Consider a typical interstellar grain, covered by a 100 monolayer (ML) thick ice. The number of sites on a grain is $10^{15} \mathrm{~cm}^{-2}$. If we assume that one in every $10^{4}$ particles on the grain is a PAH, the total number of PAH molecules on the grain is $[\mathrm{PAH}]_{0}=100 \times 10^{15} \times 10^{-4}=10^{13} \mathrm{~cm}^{-2}$. Furthermore, the VUV photon flux in our laboratory, $\Phi$, is $10^{14}$ photons $\mathrm{cm}^{-2} \mathrm{~s}^{-1}$. The production rate of PAH cations on an interstellar grain is now given by $[\mathrm{PAH}]_{0} \cdot k_{11} / \Phi=10^{-4}$ photon $^{-1}$. This ionization rate is an order of magnitude lower than the rate of photodesorption $\left(\sim 10^{-3}\right.$ photon $\left.^{-1}\right)$ (Öberg et al. 2009c).

However, in our dense cloud the number of photons available for PAH photoionization is larger than the number of photons available for photodesorption of $\mathrm{H}_{2} \mathrm{O}$ ice. This is because $\mathrm{H}_{2} \mathrm{O}$ photodesorption is primarily caused by VUV photons, whereas PAH ionization can occur for much lower energy photons. To quantify the radiation field in a dense cloud at $A_{V}=3$ as a function of wavelength $(\lambda)$, we take the average UV interstellar radiation field $\left(I_{v}\right)$ from Sternberg (1988) and rewrite the expression to $I_{\lambda}$ with units photons $\mathrm{cm}^{-2} \mathrm{~s}^{-1} \mathrm{~nm}^{-1}$

$I_{\lambda}=\frac{1.068 \times 10^{-4} c}{\lambda^{3}}-\frac{1.719 \times 10^{-2} c}{\lambda^{4}}+\frac{6.853 \times 10^{-1} c}{\lambda^{5}}$,

where $c$ is the speed of light in $\mathrm{nm} \mathrm{s}^{-1}$. The attenuation of the radiation field by dust as a function of wavelength is given by

$D_{\lambda}=\exp \left(\frac{-11.6 A_{V}}{R_{V}} \frac{A_{\lambda}}{A_{1000 \AA}}\right)$,

from Draine \& Bertoldi (1996), where we assume that $R_{V}=3.1$ and $A_{V} / A_{1000 \AA}=0.21$ (Whittet 2003). This results in

$D_{\lambda}=\exp \left(-0.8 \frac{A_{\lambda}}{A_{V}} A_{V}\right)$, where the table of $A_{\lambda} / A_{V}$ values is taken from Mathis (1990). The photon flux per second per wavelength interval is given by

$P_{\lambda}=I_{\lambda} D_{\lambda}$.

Water ice absorbs photons with wavelengths ranging from 130 to $150 \mathrm{~nm}$ (Kobayashi 1983; Andersson \& van Dishoeck 2008). The ionization energy of PAHs on the other hand, is lowered by about $2 \mathrm{eV}$ when in $\mathrm{H}_{2} \mathrm{O}$ ice (Gudipati \& Allamandola 2004; Woon \& Park 2004). For the wavelength range available for ionization of PAHs, assuming that $\mathrm{H}_{2} \mathrm{O}$ blocks all photons below $150 \mathrm{~nm}$, we take 150 to $250 \mathrm{~nm}$ (Li \& Draine 2001). By integrating the photon flux in a cloud of $A_{V}=3$ over both wavelength intervals a number of photons available for PAH ionization is found that is 6 times larger than the number of photons available for photodesorption of $\mathrm{H}_{2} \mathrm{O}$. Additionally, at $A_{V}=3$, the cosmic-ray-induced UV field is negligible compared to the interstellar UV field (Shen et al. 2004). Therefore, the occurrence of photoionization is of similar order as photodesorption of the main component in the grain mantle in a dense cloud. The ionization rates from Table 2 can be directly included in astrochemical models in the form

$\frac{\mathrm{d}\left[\mathrm{PAH}^{+}\right]}{\mathrm{d} t}=k_{11} \Psi[\mathrm{PAH}]$

where $\left[\mathrm{PAH}^{+}\right]$is the concentration of the $\mathrm{PAH}$ (pyrene) cation in the ice, $k_{11}$ is the photon rate in $\mathrm{cm}^{2}$ photon ${ }^{-1}, \Psi$ is the photon flux in photon $\mathrm{s}^{-1} \mathrm{~cm}^{2}$, and [PAH] is the PAH (pyrene) concentration in the ice.

In the above calculation, we assume that all PAHs exhibit the ionization behavior of the pyrene chromophore. Of course, more PAHs need to be investigated experimentally before drawing conclusions on their general photochemical behavior in interstellar ices. However, if all PAHs have an ionization rate similar to that of pyrene, photoionization and subsequent chemical reactions of PAHs trapped in ices are important processes in dense clouds. When frozen-out in ices, PAHs have an important impact on the radical and electron budget in solid state chemistry. Hence, the processes described here may be more important than previously assumed in modeling complex interstellar grain chemistry.

\section{Conclusions}

A recently constructed setup has been used to track, on a subsecond timescale, the photochemistry of a PAH in $\mathrm{H}_{2} \mathrm{O}$ and $\mathrm{CO}$ ices as a function of temperature. The setup used here clearly has advantages compared to relatively slow infrared photochemical ice studies. The conclusions from this work on a PAH, pyrene, trapped in an interstellar ice analogue are summarized below:

1. A set of photochemical reaction products has been identified in both irradiated $\mathrm{Py}: \mathrm{H}_{2} \mathrm{O}$ and $\mathrm{Py}: \mathrm{CO}$ ice experiments. The reaction products result from direct photoionization of pyrene, or from a reaction of the parent, pyrene, with free $\mathrm{H}$ atoms produced in the matrix. Additionally, an absorption band is tentatively assigned to a triplet-triplet transition of pyrene. A vibrational progression assigned to $\mathrm{HCO}$ is found in spectra of the VUV-irradiated Py:CO ice.

2. Pyrene is easily and efficiently ionized when trapped in $\mathrm{H}_{2} \mathrm{O}$ ice. Photoionization is a non-diffusion-related reaction and hence a photonrate of $1.2 \times 10^{-17} \mathrm{~cm}^{2}$ photon $^{-1}$, which can serve as input for astrochemical models, is derived.

3. When trapped in $\mathrm{CO}$ ice, pyrene ionization is inefficient compared to that in water ice. 
4. Electron-ion recombination is independent of ice temperature and is characterized as a non-diffusion-dominated reaction. For this process, a photon rate of $9 \times 10^{-18} \mathrm{~cm}^{2}$ photon ${ }^{-1}$ is derived, which can be directly used in astrochemical models.

5. There are two distinct reaction paths in the photochemistry of pyrene trapped in $\mathrm{H}_{2} \mathrm{O}$ ice. At low temperatures $(<50 \mathrm{~K})$, the chemistry is dominated by ion-molecule interactions and processes. At temperatures above $50 \mathrm{~K}$, reactions are dominated by diffusing radical species.

6. A simple model indicates that, in dense clouds where $A_{V}=$ 3 , the rate of pyrene ionization is comparable to the rate of photodesorption in water-rich ices. Hence, chemical reactions involving pyrene and its cation, and other PAHs in general, may be important and should be taken into account in modeling grain chemistry in these environments.

Acknowledgements. This work is financially supported by "Stichting voor Fundamenteel Onderzoek der Materie" (FOM), "the Netherlands Research School for Astronomy" (NOVA) and NASA's Laboratory Astrophysics and Astrobiology Programs. L. J. Allamandola is especially grateful to the "Nederlandse Organisatie voor Wetenschappelijk Onderzoek" (NWO) for a visitors grant.

\section{References}

Acharyya, K., Fuchs, G. W., Fraser, H. J., van Dishoeck, E. F., \& Linnartz, H. 2007, A\&A, 466, 1005

Andersson, S., \& van Dishoeck, E. F. 2008, A\&A, 491, 907

Bernstein, M. P., Sandford, S. A., Allamandola, L. J., Chang, S., \& Scharberg, M. A. 1995, ApJ, 454, 327

Bernstein, M. P., Sandford, S. A., Allamandola, L. J., et al. 1999, Science, 283, 1135

Bito, Y., Shida, N., \& Toru, T. 2000, Chem. Phys. Lett., 328, 310

Bouwman, J., Ludwig, W., Awad, Z., et al. 2007, A\&A, 476, 995

Bouwman, J., Paardekooper, D. M., Cuppen, H. M., et al. 2009, ApJ, 700, 56

Briggs, R., Ertem, G., Ferris, J. P., et al. 1992, Origins of Life and Evolution of the Biosphere, 22, 287

Draine, B. T., \& Bertoldi, F. 1996, ApJ, 468, 269

Draine, B. T., \& Li, A. 2007, ApJ, 657, 810
Draine, B. T., Dale, D. A., Bendo, G., et al. 2007, ApJ, 663, 866

Fuchs, G. W., Cuppen, H. M., Ioppolo, S., et al. 2009, A\&A, 505, 629

Gudipati, M. 2004, J. Phys. Chem. A., 108, 4412

Gudipati, M. S., \& Allamandola, L. J. 2003, ApJ, 596, L195

Gudipati, M. S., \& Allamandola, L. J. 2004, ApJ, 615, L177

Gudipati, M. S., \& Allamandola, L. J. 2006a, J. Phys. Chem. A., 110, 9020

Gudipati, M. S., \& Allamandola, L. J. 2006b, ApJ, 638, 286

Halasinski, T. M., Salama, F., \& Allamandola, L. J. 2005, ApJ, 628, 555

Hsiao, J. S., \& Webber, S. E. 1992, J. Phys. Chem., 96, 2892

Ioppolo, S., Cuppen, H. M., Romanzin, C., van Dishoeck, E. F., \& Linnartz, H. 2008, ApJ, 686, 1474

Kjaergaard, H. G., Robinson, T. W., \& Brooking, K. A. 2000, J. Phys. Chem. A, 104,11297

Kobayashi, K. 1983, J. Phys. Chem., 87, 4317

Langelaar, J., Wegdam-van Beek, J., Ten Brink, H., et al. 1970, Chem. Phys. Lett., 7, 368

Li, A., \& Draine, B. T. 2001, ApJ, 554, 778

Mathis, J. S. 1990, ARA\&A, 28, 37

Milosavljevic, B., \& Thomas, J. 2002, Photochem. Photobiol. Sci., 1, 100

Montejano, H. A., Cosa, J. J., Garrera, H. A., et al. 1995, J. Photochem. Photobiol. A: Chem., 86, 115

Muñoz Caro, G. M., Meierhenrich, U. J., Schutte, W. A., et al. 2002, Nature, 416, 403

Öberg, K. I., Fraser, H. J., Boogert, A. C. A., et al. 2007a, A\&A, 462, 1187

Öberg, K. I., Fuchs, G. W., Awad, Z., et al. 2007b, ApJ, 662, L23

Öberg, K. I., Fayolle, E. C., Cuppen, H. M., van Dishoeck, E. F., \& Linnartz, H. 2009a, A\&A, 505, 183

Öberg, K. I., Garrod, R. T., van Dishoeck, E. F., et al. 2009b, A\&A, 504, 891

Öberg, K. I., Linnartz, H., Visser, R., et al. 2009c, ApJ, 693, 1209

Okada, T., Mori, T., \& Mataga, N. 1976, Bull. Chem. Soc. Japan, 49, 3398

Okada, T., Tashita, N., \& Mataga, N. 1980, Chem. Phys. Lett., 75, 220

Shen, C. J., Greenberg, J. M., Schutte, W. A., et al. 2004, A\&A, 415, 203

Smith, J. D. T., Draine, B. T., Dale, D. A., et al. 2007, ApJ, 656, 770

Sternberg, A. 1988, ApJ, 332, 400

Stevens, B., Thomaz, M. F., \& Jones, J. 1967, J. Chem. Phys., 46, 405

Tielens, A. G. G. M. 2008, ARA\&A, 46, 289

Vala, M., Szczepanski, J., Pauzat, F., et al. 1994, J. Phys. Chem., 98, 9187

van Ijzendoorn, L. J., Allamandola, L. J., Baas, F., et al. 1983, J. Chem. Phys., 78,7019

Wang, B. C., Chang, J. C., Tso, H. C., Hsu, H. F., \& Cheng, C. Y. 2003, J. Mol. Struct. (Theochem), 629, 11

Weisman, J. L., Mattioda, A., Lee, T. J., et al. 2005, Phys. Chem. Chem. Phys. (Incorporating Faraday Transactions), 7, 109

Whittet, D. C. B. 2003, Dust in the galactic environment (Bristol: IOP Publ.)

Whittet, D. C. B., Gerakines, P. A., Hough, J. H., et al. 2001, ApJ, 547, 872

Woon, D. E., \& Park, J.-Y. 2004, ApJ, 607, 342 